\title{
Verifying Communicating Agents by Model Checking in a Temporal Action Logic
}

\author{
Laura Giordano ${ }^{1}$, Alberto Martelli ${ }^{2}$, Camilla Schwind ${ }^{3}$ \\ ${ }^{1}$ Dipartimento di Informatica, Università del Piemonte Orientale, Alessandria \\ ${ }^{2}$ Dipartimento di Informatica, Università di Torino, Torino \\ ${ }^{3}$ MAP, CNRS, Marseille, France
}

\begin{abstract}
In this paper we address the problem of specifying and verifying systems of communicating agents in a Dynamic Linear Time Temporal Logic (DLTL). This logic provides a simple formalization of the communicative actions in terms of their effects and preconditions. Furthermore it allows to specify interaction protocols by means of temporal constraints representing permissions and commitments. Agent programs, when known, can be formulated in DLTL as complex actions (regular programs). The paper addresses several kinds of verification problems including the problem of compliance of agents to the protocol, and describes how they can be solved by model checking in DLTL using automata.
\end{abstract}

\section{Introduction}

The specification and the verification of the behavior of interacting agents is one of the central issues in the area of multi-agent systems. In this paper we address the problem of specifying and verifying systems of communicating agents in a Dynamic Linear Time Temporal Logic (DLTL).

The extensive use of temporal logics in the specification and verification of distributed systems has led to the development of many techniques and tools for automating the verification task. Recently, temporal logics have gained attention in the area of reasoning about actions and planning $[2,10,12,17,5]$, and they have also been used in the specification and in the verification of systems of communicating agents. In particular, in [21] agents are written in MABLE, an imperative programming language, and the formal claims about the system are expressed using a quantified linear time temporal BDI logic and can be automatically verified by making use of the SPIN model checker. Guerin in [13] defines an agent communication framework which gives agent communication a grounded declarative semantics. In such a framework, temporal logic is used for formalizing temporal properties of the system.

In this paper we present a theory for reasoning about communicative actions in a multiagent system which is based on the Dynamic Linear Time Temporal

\footnotetext{
* This research has been partially supported by the project PRIN 2003 "Logic-based development and verification of multi-agent systems", and by the European Commission within the 6th Framework Programme project REWERSE number 506779
} 
Logic $(D L T L)$ [15], which extends LTL by strengthening the until operator by indexing it with the regular programs of dynamic logic. As a difference with [21] we adopt a social approach to agent communication $[1,7,19,13]$, in which communicative actions affect the "social state" of the system, rather than the internal (mental) states of the agents. The social state records social facts, like the permissions and the commitments of the agents. The dynamics of the system emerges from the interactions of the agents, which must respect these permissions and commitments (if they are compliant with the protocol). The social approach allows a high level specification of the protocol, and does not require the rigid specification of the allowed action sequences. It is well suited for dealing with "open" multiagent systems, where the history of communications is observable, but the internal states of the single agents may not be observable.

Our proposal relies on the theory for reasoning about action developed in [10] which is based on DLTL and which allows reasoning with incomplete initial states and dealing with postdiction, ramifications as well as with nondeterministic actions. It allows a simple formalization of the communicative actions in terms of their effects and preconditions as well as the specification of an interaction protocol to constrain the behaviors of autonomous agents.

In [11] we have presented a proposal for reasoning about communicating agents in the Product Version of DLTL, which allows to describe the behavior of a network of sequential agents which coordinate their activities by performing common actions together. Here we focus on the non-product version of DLTL, which appears to be a simpler choice and also a more reasonable choice when a social approach is adopted. In fact, the Product Version of DLTL does not allow to describe global properties of a system of agents, as it keeps the local states of the agents separate. Instead, the "social state" of the system is inherently global and shared by all of the agents. Moreover, we will see that the verification tasks described in [11] can be conveniently represented in DLTL without requiring the product version. The verification of the compliance of an agent to the protocol, the verification of protocol properties, the verification that an agent is (is not) respecting its social facts (commitments and permissions) at runtime are all examples of tasks which can be formalized either as validity or as satisfiability problems in DLTL. Such verification tasks can be automated by making use of Büchi automata. In particular, we make use of the tableau-based algorithm presented in [9] for constructing a Büchi automaton from a DLTL formula. The construction of the automata can be done on-the-fly, while checking for the emptiness of the language accepted by the automaton. As for LTL, the number of states of the automata is, in the worst case, exponential in the size of the input formula.

\section{Dynamic Linear Time Temporal Logic}

In this section we shortly define the syntax and semantics of DLTL as introduced in [15]. In such a linear time temporal logic the next state modality is indexed 
by actions. Moreover, (and this is the extension to LTL) the until operator is indexed by programs in Propositional Dynamic Logic (PDL).

Let $\Sigma$ be a finite non-empty alphabet. The members of $\Sigma$ are actions. Let $\Sigma^{*}$ and $\Sigma^{\omega}$ be the set of finite and infinite words on $\Sigma$, where $\omega=\{0,1,2, \ldots\}$. Let $\Sigma^{\infty}=\Sigma^{*} \cup \Sigma^{\omega}$. We denote by $\sigma, \sigma^{\prime}$ the words over $\Sigma^{\omega}$ and by $\tau, \tau^{\prime}$ the words over $\Sigma^{*}$. Moreover, we denote by $\leq$ the usual prefix ordering over $\Sigma^{*}$ and, for $u \in \Sigma^{\infty}$, we denote by $\operatorname{prf}(u)$ the set of finite prefixes of $u$.

We define the set of programs (regular expressions) $\operatorname{Prg}(\Sigma)$ generated by $\Sigma$ as follows:

$$
\operatorname{Prg}(\Sigma)::=a\left|\pi_{1}+\pi_{2}\right| \pi_{1} ; \pi_{2} \mid \pi^{*}
$$

where $a \in \Sigma$ and $\pi_{1}, \pi_{2}, \pi$ range over $\operatorname{Prg}(\Sigma)$. A set of finite words is associated with each program by the mapping [[]] $\operatorname{Prg}(\Sigma) \rightarrow 2^{\Sigma^{*}}$, which is defined as follows:

$$
\begin{aligned}
& -[[a]]=\{a\} ; \\
& -\left[\left[\pi_{1}+\pi_{2}\right]\right]=\left[\left[\pi_{1}\right]\right] \cup\left[\left[\pi_{2}\right]\right] ; \\
& -\left[\left[\pi_{1} ; \pi_{2}\right]\right]=\left\{\tau_{1} \tau_{2} \mid \tau_{1} \in\left[\left[\pi_{1}\right]\right] \text { and } \tau_{2} \in\left[\left[\pi_{2}\right]\right]\right\} ; \\
& -\left[\left[\pi^{*}\right]\right]=\bigcup\left[\left[\pi^{i}\right]\right], \text { where } \\
& \quad \bullet\left[\left[\pi^{0}\right]\right]=\{\varepsilon\} \\
& \quad \bullet\left[\left[\pi^{i+1}\right]\right]=\left\{\tau_{1} \tau_{2} \mid \tau_{1} \in[[\pi]] \text { and } \tau_{2} \in\left[\left[\pi^{i}\right]\right]\right\}, \text { for every } i \in \omega .
\end{aligned}
$$

Let $\mathcal{P}=\left\{p_{1}, p_{2}, \ldots\right\}$ be a countable set of atomic propositions. The set of formulas of $\operatorname{DLTL}(\Sigma)$ is defined as follows:

$$
\operatorname{DLTL}(\Sigma)::=p|\neg \alpha| \alpha \vee \beta \mid \alpha \mathcal{U}^{\pi} \beta
$$

where $p \in \mathcal{P}$ and $\alpha, \beta$ range over $\operatorname{DLTL}(\Sigma)$.

A model of $\operatorname{DLTL}(\Sigma)$ is a pair $M=(\sigma, V)$ where $\sigma \in \Sigma^{\omega}$ and $V: \operatorname{prf}(\sigma) \rightarrow$ $2^{\mathcal{P}}$ is a valuation function. Given a model $M=(\sigma, V)$, a finite word $\tau \in \operatorname{prf}(\sigma)$ and a formula $\alpha$, the satisfiability of a formula $\alpha$ at $\tau$ in $M$, written $M, \tau=\alpha$, is defined as follows:

$-M, \tau \models p$ iff $p \in V(\tau)$;

$-M, \tau \models \neg \alpha$ iff $M, \tau \mid \models \alpha$

$-M, \tau \models \alpha \vee \beta$ iff $M, \tau=\alpha$ or $M, \tau \models \beta$;

$-M, \tau \models \alpha \mathcal{U}^{\pi} \beta$ iff there exists $\tau^{\prime} \in[[\pi]]$ such that $\tau \tau^{\prime} \in \operatorname{prf}(\sigma)$ and $M, \tau \tau^{\prime} \models$ $\beta$. Moreover, for every $\tau^{\prime \prime}$ such that $\varepsilon \leq \tau^{\prime \prime}<\tau^{\prime 1}, M, \tau \tau^{\prime \prime} \models \alpha$.

A formula $\alpha$ is satisfiable iff there is a model $M=(\sigma, V)$ and a finite word $\tau \in \operatorname{prf}(\sigma)$ such that $M, \tau \models \alpha$.

The formula $\alpha \mathcal{U}^{\pi} \beta$ is true at $\tau$ if " $\alpha$ until $\beta$ " is true on a finite stretch of behavior which is in the linear time behavior of the program $\pi$.

The derived modalities $\langle\pi\rangle$ and $[\pi]$ can be defined as follows: $\langle\pi\rangle \alpha \equiv \top \mathcal{U}^{\pi} \alpha$ and $[\pi] \alpha \equiv \neg\langle\pi\rangle \neg \alpha$.

\footnotetext{
$\overline{{ }^{1} \text { We define } \tau} \leq \tau^{\prime}$ iff $\exists \tau^{\prime \prime}$ such that $\tau \tau^{\prime \prime}=\tau^{\prime}$. Moreover, $\tau<\tau^{\prime}$ iff $\tau \leq \tau^{\prime}$ and $\tau \neq \tau^{\prime}$.
} 
Furthermore, if we let $\Sigma=\left\{a_{1}, \ldots, a_{n}\right\}$, the $\mathcal{U}, O$ (next), $\diamond$ and $\square$ operators of LTL can be defined as follows: $O \alpha \equiv \bigvee_{a \in \Sigma}\langle a\rangle \alpha, \alpha \mathcal{U} \beta \equiv \alpha \mathcal{U}^{\Sigma^{*}} \beta, \diamond \alpha \equiv \top \mathcal{U} \alpha$, $\square \alpha \equiv \neg \diamond \neg \alpha$, where, in $\mathcal{U}^{\Sigma^{*}}, \Sigma$ is taken to be a shorthand for the program $a_{1}+\ldots+a_{n}$. Hence both $\operatorname{LTL}(\Sigma)$ and PDL are fragments of $\operatorname{DLTL}(\Sigma)$. As shown in [15], DLTL $(\Sigma)$ is strictly more expressive than $\operatorname{LTL}(\Sigma)$. In fact, DLTL has the full expressive power of the monadic second order theory of $\omega$-sequences.

\section{Action theories}

In this section we recall the action theory developed in [10] that we use for specifying the interaction between communicating agents.

Let $\mathcal{P}$ be a set of atomic propositions, the fluent names. A fluent literal $l$ is a fluent name $f$ or its negation $\neg f$. Given a fluent literal $l$, such that $l=f$ or $l=\neg f$, we define $|l|=f$. We will denote by Lit the set of all fluent literals.

A domain description $D$ is defined as a tuple $(\Pi, \mathcal{C})$, where $\Pi$ is a set of action laws and causal laws, and $\mathcal{C}$ is a set of constraints.

Action laws in $\Pi$ have the form: $\square(\alpha \rightarrow[a] \beta)$, with $a \in \Sigma$ and $\alpha, \beta$ arbitrary formulas, meaning that executing action $a$ in a state where precondition $\alpha$ holds causes the effect $\beta$ to hold.

Causal laws in $\Pi$ have the form: $\square((\alpha \wedge \bigcirc \beta) \rightarrow \bigcirc \gamma)$, meaning that if $\alpha$ holds in a state and $\beta$ holds in the next state, then $\gamma$ also holds in the next state. Such laws are intended to expresses "causal" dependencies among fluents.

Constraints in $\mathcal{C}$ are arbitrary temporal formulas of $D L T L$. In particular, the set of constraints $\mathcal{C}$ contains all the temporal formulas which might be needed to constrain the behaviour of a protocol, including the value of fluents in the initial state. The set of constraints $\mathcal{C}$ also includes the precondition laws.

Precondition laws have the form: $\square(\alpha \rightarrow[a] \perp)$, meaning that the execution of an action $a$ is not possible if $\alpha$ holds (i.e. there is no resulting state following the execution of $a$ if $\alpha$ holds). Observe that, when there is no precondition law for an action, the action is executable in all states.

Action laws and causal laws describe the changes to the state. All other fluents which are not changed by the actions are assumed to persist unaltered to the next state. To cope with the frame problem, the laws in $\Pi$, describing the (immediate and ramification) effects of actions, have to be distinguished from the constraints in $\mathcal{C}$ and given a special treatment. In [10], to deal with the frame problem, a completion construction is defined which, given a domain description, introduces frame axioms for all the frame fluents in the style of the successor state axioms introduced by Reiter [18] in the context of the situation calculus. The completion construction is applied only to the action laws and causal laws in $\Pi$ and not to the constraints. In the following we call $\operatorname{Comp}(\Pi)$ the completion of a set of laws $\Pi$ and we refer to [10] for the details on the completion construction.

Test actions allow the choice among different behaviours to be controlled. As DLTL does not include test actions, we introduce them in the language as atomic actions in the same way as done in [10]. More precisely, we introduce 
an atomic action $\phi$ ? for each proposition $\phi$ we want to test. The test action $\phi$ ? is executable in any state in which $\phi$ holds and it has no effect on the state. Therefore, we introduce the following laws which rule the modality $[\phi ?]$ :

$$
\begin{aligned}
& \square(\neg \phi \rightarrow[\phi ?] \perp) \\
& \square(\langle\phi ?\rangle \top \rightarrow(L \leftrightarrow[\phi ?] L)), \text { for all fluent literals } L .
\end{aligned}
$$

The first law is a precondition law, saying that action $\phi$ ? is only executable in a state in which $\phi$ holds. The second law describes the effects of the action on the state: the execution of the action $\phi$ ? leaves the state unchanged. We assume that, for all test actions occurring in a domain description, the corresponding action laws are implicitly added.

As a difference from [10], in this paper we will use, besides boolean fluents, functional fluents, i.e. fluents which take a value in a (finite) set. We use the notation $f=V$ to say that fluent $f$ has value $V$. It is clear however, that functional fluents can be easily represented by making use of multiple (and mutually exclusive) boolean fluents.

\section{Contract Net Protocol}

In the social approach $[7,13,19,22]$ an interaction protocol is specified by describing the effects of communicative actions on the social state, and by specifying the permissions and the commitments that arise as a result of the current conversation state. In our action theory the effects of communicative actions will be modelled by action laws. Permissions, which determine when an action can be taken by each agent, can be modelled by precondition laws. Commitment policies, which rule the dynamic of commitments, can be described by causal laws which establish the causal dependencies among fluents. The specification of a protocol can be further constrained through the addition of suitable temporal formulas, and also the agents' programs can be modelled, by making use of complex actions (regular programs).

As a running example we will use the Contract Net protocol [6].

Example 1. The Contract Net protocol begins with an agent (the manager) broadcasting a task announcement (call for proposals) to other agents viewed as potential contractors (the participants). Each participant can reply by sending either a proposal or a refusal. The manager must send an accept or reject message to all those who sent a proposal. When a contractor receives an acceptance it is committed to perform the task. For lack of space we will leave out the final step of the protocol.

Let us consider first the simplest case where we have only two agents: the manager $(\mathrm{M})$ and the participant $(\mathrm{P})$. The two agents share all the communicative actions, which are: $c f p(T)$ (the manager issues a call for proposals for task $\mathrm{T})$, accept and reject whose sender is the manager, and refuse and propose whose sender is the participant. 
The social state will contain the following domain specific fluents: task (a functional fluent whose value is the task which has been announced, or nil if the task has not yet been announced), replied (the participant has replied), proposal (the participant has sent a proposal) and acc_rej (the manager has sent an accept or reject message). Such fluents describe observable facts concerning the execution of the protocol.

We also introduce special fluents to represent base-level commitments of the form $C(i, j, \alpha)$, meaning that agent $i$ is committed to agent $j$ to bring about $\alpha$, where $\alpha$ is an arbitrary formula, or they can be conditional commitments of the form $C C(i, j, \beta, \alpha)$ (agent $i$ is committed to agent $j$ to bring about $\alpha$, if the condition $\beta$ is brought about $)^{2}$. For modelling the Contract Net example we introduce the following commitments

$$
C(P, M \text {, replied }) \text { and } C(M, P, \text { acc_rej })
$$

and conditional commitments

$$
C C(P, M, \text { task } \neq \text { nil, replied }) \text { and } C C(M, P \text {, proposal,acc_rej }) \text {. }
$$

Some reasoning rules have to be defined for cancelling commitments when they have been fulfilled and for dealing with conditional commitments. We introduce the following causal laws:

$$
\begin{aligned}
& \square(\bigcirc \alpha \rightarrow \bigcirc \neg C(i, j, \alpha)) \\
& \square(\bigcirc \alpha \rightarrow \bigcirc \neg C C(i, j, \beta, \alpha)) \\
& \square((C C(i, j, \beta, \alpha) \wedge \bigcirc \beta) \rightarrow \bigcirc(C(i, j, \alpha) \wedge \neg C C(i, j, \beta, \alpha)))
\end{aligned}
$$

A commitment (or a conditional commitment) to bring about $\alpha$ is cancelled when $\alpha$ holds, and a conditional commitment $C C(i, j, \beta, \alpha)$ becomes a base-level commitment $C(i, j, \alpha)$ when $\beta$ has been brought about.

Let us now describe the effects of communicative actions by the following action laws:

$$
\begin{aligned}
& \square[\text { cfp }(T)] \text { task }=T \\
& \square[\text { cfp }(T)] C C(M, P, \text { proposal }, \text { acc_rej }) \\
& \square[\text { accept }] \text { acc_rej } \\
& \square[\text { reject }] \text { acc_rej } \\
& \square[\text { refuse }] \text { replied } \\
& \square[\text { propose }](\text { replied } \wedge \text { proposal })
\end{aligned}
$$

The laws for action $c f p(T)$ add to the social state the information that a call for proposal has been done for the task $T$, and that, if the manager receives a proposal, it is committed to accept or reject it.

The permissions to execute communicative actions in each state are determined by social facts. We represent them by precondition laws. Preconditions on the execution of action accept can be expressed as:

\footnotetext{
2 The two kinds of base-level and conditional commitments we allow are essentially those introduced in [22]. Such choice is different from the one in [13] and in [11], where agents are committed to execute an action rather than to achieve a condition.
} 
$\square(\neg$ proposal $\vee$ acc_rej $\rightarrow[$ accept $] \perp)$

meaning that action accept cannot be executed only if a proposal has not been done, or if the manager has already replied. Similarly we can give the precondition laws for the other actions:

$$
\begin{aligned}
& \square(\neg \text { proposal } \vee \text { acc_re } \rightarrow[\text { reject }] \perp) \\
& \square(\text { task }=\text { nil } \vee \text { replied } \rightarrow[\text { refuse }] \perp) \\
& \square(\text { task }=n i l \vee \text { replied } \rightarrow[\text { propose }] \perp) \\
& \square(\text { task } \neq n i l \rightarrow[\text { cfp }(T)] \perp) .
\end{aligned}
$$

The precondition law for action propose (refuse) says that a proposal can only be done if task $\neq$ nil, that is, if a task has already been announced and the participant has not already replied. The last law says that the manager cannot issue a new call for proposal if task $\neq n i l$, that is, if a task has already been announced.

In the following we will denote $\operatorname{Perm}_{i}$ (permissions of agent i) the set of all the precondition laws of the protocol pertaining to the actions of which agent $i$ is the sender.

Assume now that we want the participant to be committed to reply to the task announcement. We can express it by adding the following conditional commitment to the initial state of the protocol: $C C(P, M$, task $\neq$ nil, replied $)$. Furthermore the manager is committed initially to issue a call for proposal for a task. We can define the initial state Init of the protocol as follows:

$$
\begin{aligned}
& \{\text { task }=\text { nil }, \neg \text { replied }, \neg \text { proposal, } C C(P, M, \text { task } \neq \text { nil, replied }), \\
& C(M, P, \text { task } \neq \text { nil })\}
\end{aligned}
$$

In the following we will be interested in those execution of the protocol in which all commitments have been fulfilled. We can express the condition that the commitment $C(i, j, \alpha)$ will be fulfilled by the following constraint:

$$
\square(C(i, j, \alpha) \rightarrow \diamond \alpha)
$$

We will call $\mathrm{Com}_{i}$ the set of constraints of this kind for all commitments of agent $i$. Com $i$ states that agent $i$ will fulfill all the commitments of which he is the debtor.

Given the above rules, the domain description $D=(\Pi, \mathcal{C})$ of a protocol is defined as follows: $\Pi$ is the set of the action and causal laws given above, and $\mathcal{C}=$ Init $\wedge \bigwedge_{i}\left(\right.$ Perm $\left._{i} \wedge \mathrm{Com}_{i}\right)$ is the set containing the constraints on the initial state, the permissions Perm $_{i}$ and the commitments Com $_{i}$ of all the agents (the agents $\mathrm{P}$ and $\mathrm{M}$, in this example).

Given a domain description $D$, let the completed domain description $\operatorname{Comp}(D)$ be the set of formulas $\left(\operatorname{Comp}(\Pi) \wedge\right.$ Init $\left.\wedge \bigwedge_{i}\left(\operatorname{Perm}_{i} \wedge \operatorname{Com}_{i}\right)\right)$. The runs of the system according the protocol are the linear models of $\operatorname{Comp}(D)$. Observe that in these protocol runs all permissions and commitments have been fulfilled. However, if $\mathrm{Com}_{j}$ is not included for some agent $j$, the runs may contain commitments which have not been fulfilled by $j$. 


\section{Verification}

Given the DLTL specification of a protocol by a domain description, we describe the different kinds of verification problems which can be addressed.

First, given an execution history describing the interactions of the agents, we want to verify the compliance of that execution to the protocol. This verification is carried out at runtime. We are given a history $\tau=a_{1}, \ldots, a_{n}$ of the communicative actions executed by the agents, and we want to verify that the history $\tau$ is the prefix of a run of the protocol, that is, it respects the permissions and commitments of the protocol. This problem can be formalized by requiring that the formula

$$
\left(\operatorname{Comp}(\Pi) \wedge \operatorname{Init} \wedge \bigwedge_{i}\left(\operatorname{Perm}_{i} \wedge \operatorname{Com}_{i}\right)\right) \wedge<a_{1} ; a_{2} ; \ldots ; a_{n}>\top
$$

(where $i$ ranges on all the agents involved in the protocol) is satisfiable. In fact, the above formula is satisfiable if it is possible to find a run of the protocol starting with the action sequence $a_{1}, \ldots, a_{n}$.

A second problem is that of proving a property $\varphi$ of a protocol. This can be formulated as the validity of the formula

$$
\left(\operatorname{Comp}(\Pi) \wedge \operatorname{Init} \wedge \bigwedge_{i}\left(\operatorname{Perm}_{i} \wedge \operatorname{Com}_{i}\right)\right) \rightarrow \varphi .
$$

Observe that, to prove the property $\varphi$, all the agents are assumed to be compliant with the protocol.

A further problem is to verify that an agent is compliant with the protocol, given the program executed by the agent itself. In our formalism we can specify the behavior of an agent by making use of complex actions (regular programs). Consider for instance the following program $\pi_{P}$ for the participant:

$[\neg$ done?; $((c f p(T) ;$ eval_task; $(\neg o k ? ;$ refuse $;$ exit + ok?; propose $))+$ $($ reject $;$ exit $)+($ accept; do_task; exit $))]^{*} ;$ done?

The participant cycles and reacts to the messages received by the manager: for instance, if the manager has issued a call for proposal, the participant can either refuse or make a proposal according to his evaluation of the task; if the manager has accepted the proposal, the participant performs the task; and so on.

The state of the agent is obtained by adding to the fluents of the protocol the following local fluents: done, which is initially false and is made true by action exit, and $o k$ which says if the agent must make a bid or not. The local actions are eval_task, which evaluates the task and sets the fluent $o k$ to true or false, do_task and exit. Furthermore, done? and $o k$ ? are test actions.

The program of the contractor can be specified by a domain description $\operatorname{Prog}_{P}=\left(\Pi_{P}, \mathcal{C}_{P}\right)$, where $\Pi_{P}$ is a set of action laws describing the effects of the private actions of the contractor, for instance: 


$$
\begin{aligned}
& \square[\text { exit }] \text { done } \\
& \square(\text { task }=t 1 \rightarrow[\text { eval_task }] \text { ok }) \\
& \square(\text { task }=t 2 \rightarrow[\text { eval_task }] \neg o k)
\end{aligned}
$$

and, $\mathcal{C}_{P}=\left\{\left\langle\pi_{P}\right\rangle \top, \neg\right.$ done,$\left.\neg o k\right\}$ contains the constraints on the initial values of fluents $(\neg$ done, $\neg o k)$ as well as the formula $\left\langle\pi_{P}\right\rangle \top$ stating that the program of the participant is executable in the initial state.

We want now to prove that the participant is compliant with the protocol, i.e. that all executions of program $\pi_{P}$ satisfy the specification of the protocol. This property cannot be proved by considering only the program $\pi_{P}$. In fact, it is easy to see that the correctness of the property depends on the behavior of the manager. For instance, if the manager begins with an accept action, the participant will execute the sequence of actions accept;do_task; exit and stop, which is not a correct execution of the protocol. Thus we have to take into account also the behavior of the manager. Since we don't know its internal behavior, we will assume that the manager respects its public behavior, i.e. that it respects its permissions and commitments in the protocol specification.

The verification that the participant is compliant with the protocol can be formalized as a validity check. Let $D=(\Pi, \mathcal{C})$ be the domain description describing the protocol, as defined above. The formula

$\left(\operatorname{Comp}(\Pi) \wedge \operatorname{Init} \wedge \operatorname{Perm}_{M} \wedge \operatorname{Com}_{M} \wedge \operatorname{Comp}\left(\Pi_{P}\right) \wedge \mathcal{C}_{P}\right) \rightarrow\left(\operatorname{Perm}_{P} \wedge \operatorname{Com}_{P}\right)$

is valid if in all the behaviors of the system, in which the participant executes its program $\pi_{P}$ and the manager (whose internal program is unknown) respects the protocol specification (in particular, its permissions and commitments), the permissions and commitment of the participant are also satisfied.

\section{Contract Net with $N$ participants}

Let us assume now that we have $N$ potential contractors. The above formulation of the protocol can be extended by introducing a fluent $\operatorname{replied}(i), \operatorname{proposal}(i)$ and $a c c \_r e j(i)$ for each participant $i$, and similarly for the commitments. Furthermore we introduce the communicative actions refuse $(i)$, and propose $(i)$, which are sent from participant $i$ to the manager, and reject $(i)$ and accept $(i)$, which are sent from the manager to participant $i$. We assume action $c f p(T)$ to be shared by all agents (broadcast by the manager).

The theory describing the new version of the protocol can be easily obtained from the one given above. For instance the precondition laws for accept $(i)$ and reject $(i)$ must be modified so that these actions will be executed only after all participants have replied to the manager, i.e.:

$$
\square\left(\left(\neg \text { proposal }(i) \vee \text { acc_rej }(i) \vee \bigvee_{j=1, N} \neg \operatorname{replied}(j)\right) \rightarrow[\operatorname{accept}(i)] \perp\right)
$$

and the same for reject $(i)$.

The verification problems mentioned before can be formulated using the same approach. For instance, the verification that the protocol satisfies a given property $\varphi$ can be expressed as the validity of the formula (1) above, where $i$ ranges 
over the $N$ participants and the manager. To prove compliance of a participant with the protocol, we need to restrict the protocol to the actions and the fluents shared between the manager and this participant (i.e. we need to take the projection of the protocol on agent $i$ and the agents with whom $i$ interacts). Then the problem can be formulated as in the case of a single participant.

We have not considered here the formulation of the problem in which proposals must be submitted within a given deadline. This would require adding to the system a further agent clock.

\section{$7 \quad$ Model checking}

The above verification and satisfiability problems can be solved by extending the standard approach for verification and model-checking of Linear Time Temporal Logic, based on the use of Büchi automata. As described in [15], the satisfiability problem for DLTL can be solved in deterministic exponential time, as for LTL, by constructing for each formula $\alpha \in D L T L(\Sigma)$ a Büchi automaton $\mathcal{B}_{\alpha}$ such that the language of $\omega$-words accepted by $\mathcal{B}_{\alpha}$ is non-empty if and only if $\alpha$ is satisfiable. Actually a stronger property holds, since there is a one to one correspondence between models of the formula and infinite words accepted by $\mathcal{B}_{\alpha}$. The size of the automaton can be exponential in the size of $\alpha$, while emptiness can be detected in a time linear in the size of the automaton.

The validity of a formula $\alpha$ can be verified by constructing the Büchi automaton $\mathcal{B}_{\neg \alpha}$ for $\neg \alpha$ : if the language accepted by $\mathcal{B}_{\neg \alpha}$ is empty, then $\alpha$ is valid, whereas any infinite word accepted by $\mathcal{B}_{\neg \alpha}$ provides a counterexample to the validity of $\alpha$.

For instance, let $\mathrm{CN}$ be the completed domain description of the Contract Net protocol, that is $C N=\left(\operatorname{Comp}(\Pi) \wedge\right.$ Init $\left.\wedge \bigwedge_{i}\left(\operatorname{Perm}_{i} \wedge \operatorname{Com}_{i}\right)\right)$. Then every infinite word accepted by $\mathcal{B}_{C N}$ corresponds to a possible execution of the protocol. To prove a property $\varphi$ of the protocol, we can build the automaton $\mathcal{B}_{\neg \varphi}$ and check that the language accepted by the product of $\mathcal{B}_{C N}$ and $\mathcal{B}_{\neg \varphi}$ is empty.

The construction given in [15] is highly inefficient since it requires to build an automaton with an exponential number of states, most of which will not be reachable from the initial state. A more efficient approach for constructing a Büchi automaton from a DLTL formula makes use of a tableau-based algorithm [9]. The construction of the states of the automaton is similar to the standard construction for LTL [8], but the possibility of indexing until formulas with regular programs puts stronger constraints on the fulfillment of until formulas than in LTL, requiring more complex acceptance conditions. The construction of the automaton can be done on-the-fly, while checking for the emptiness of the language accepted by the automaton. Given a formula $\varphi$, the algorithm builds a graph $\mathcal{G}(\varphi)$ whose nodes are labelled by sets of formulas. States and transitions of the Büchi automaton correspond to nodes and arcs of the graph. The algorithm makes use of an auxiliary tableau-based function which expands the set of formulas at each node. As for LTL, the number of states of the automaton is, 
in the worst case, exponential in the size if the input formula, but in practice it is much smaller. For instance, the automaton obtained from the Contract Net protocol has about 20 states.

LTL is widely used to prove properties of (possibly concurrent) programs by means of model checking techniques. The property is represented as an LTL formula $\varphi$, whereas the program generates a Kripke structure (the model), which directly corresponds to a Büchi automaton where all the states are accepting, and which describes all possible computations of the program. The property can be proved as before by taking the product of the model and of the automaton derived from $\neg \varphi$, and by checking for emptiness of the accepted language.

In principle, with DLTL we do not need to use model checking, because programs and domain descriptions can be represented in the logic itself, as we have shown in the previous section. However representing everything as a logical formula can be rather inefficient from a computational point of view. In particular all formulas of the domain description are universally quantified, and this means that our algorithm will have to propagate them from each state to the next one, and to expand them with the tableau procedure at each step.

Therefore we have adapted model checking to the proof of the formulas given in the previous section, as follows. Let us assume that the negation of a formula to be proved can be represented as $F \wedge \varphi$, where $F=\operatorname{Comp}(\Pi) \wedge$ Init contains the completion of the action and causal laws in the domain description and the initial state, and $\varphi$ the rest of the formula. For instance, in the verification of the compliance of the participant, the negation of the formula to be proved is $\left(\operatorname{Comp}(\Pi) \wedge\right.$ Init $\left.\wedge \operatorname{Perm}_{M} \wedge \operatorname{Com}_{M} \wedge \operatorname{Comp}\left(\Pi_{P}\right) \wedge \neg\left(\operatorname{Perm}_{P} \wedge \operatorname{Com}_{P}\right)\right)$ and thus $\varphi=\left(\operatorname{Perm}_{M} \wedge \operatorname{Com}_{M} \wedge \operatorname{Comp}\left(\Pi_{P}\right) \wedge \neg\left(\operatorname{Perm}_{P} \wedge \operatorname{Com}_{P}\right)\right)$. We can derive from $F$ an automaton describing all possible computations, whose states are sets of fluents, which we consider as the model. In particular, we can obtain from the domain description a function $\operatorname{trans}_{a}(S)$, for each action $a$, for transforming a state in the next one, and then build this automaton by repeatedly applying these functions starting from the initial state. We can then proceed by taking the product of the model and of the automaton derived from $\varphi$, and by checking for emptiness of the accepted language.

Note that, although this automaton has an exponential number of states, we can build it step by step by following the construction of the algorithm on-thefly. The state of the product automaton will consist of two parts $<S_{1}, S_{2}>$, where $S_{1}$ is a set of fluents representing a state of the model, and $S_{2}$ is a set of formulas. The initial state will be $\langle I, \varphi\rangle$, where $I$ is the initial set of fluents. A successor state through a transition $a$ will be obtained as $<\operatorname{trans}_{a}\left(S_{1}\right), S_{2}^{\prime}>$ where $S_{2}^{\prime}$ is derived from $S_{2}$ by the on-the-fly algorithm. If the two parts of a state are inconsistent, the state is discarded.

\section{Conclusions}

We have shown that DLTL is a suitable formalism for specifying and verifying a system of communicating agents. Our approach provides a unified framework for 
describing different aspects of multi-agent systems. Programs are expressed as regular expressions, (communicative) actions can be specified by means of action and precondition laws, properties of social facts can be specified by means of causal laws and constraints, and temporal properties can be expressed by means of the until operator. We have addressed several kinds of verification problems, including the problem of compliance of agents to the protocol, and described how they can be solved by developing automata-based model checking techniques for DLTL. A preliminary implementation of a model checker based on the algorithm in [9] is being tested in the verification of the properties of various protocols.

The issue of developing semantics for agent communication languages has been examined in [20], by considering in particular the problem of giving a verifiable semantics, i.e. a semantics grounded on the computational models. Guerin and Pitt $[13,14]$ define an agent communication framework which gives agent communication a grounded declarative semantics. The framework introduces different languages: a language for agent programming, a language for specifying agent communication and social facts, and a language for expressing temporal properties. Our approach instead provides a unified framework for describing multiagent systems using DLTL.

While in this paper we follow a social approach to the specification and verification of systems of communicating agents, [4, 3, 16, 21] have adopted a mentalistic approach. The goal of [3] is to extend model checking to make it applicable to multi-agent systems, where agents have BDI attitudes. This is achieved by using a new logic which is the composition of two logics, one formalizing temporal evolution and the other formalizing BDI attitudes. In $[16,21]$ agents are written in MABLE, an imperative programming language, and have a mental state. MABLE systems may be augmented by the addition of formal claims about the system, expressed using a quantified, linear time temporal BDI logic. Instead [4] deals with programs written in $\operatorname{AgentSpeak}(\mathrm{F})$, a variation of the BDI logic programming language AgentSpeak(L). Properties of MABLE or AgentSpeak programs can be verified by means of the SPIN model checker. These papers do not deal with the problem of proving properties of protocols.

Yolum and Singh [22] developed a social approach to protocol specification and execution. In this approach, commitments are formalized in a variant of event calculus. By using an event calculus planner it is possible to determine execution paths that respect the protocol specification. Alberti et al. address a similar problem, by expressing protocols in a logic-based formalism based on Social Integrity Constraints. In [1] they present a system that, during the evolution of a society of agents, verifies the compliance of the agents' behavior to the protocol.

\section{References}

1. M. Alberti, D. Daolio and P. Torroni. Specification and Verification of Agent Interaction Protocols in a Logic-based System. SAC'04, March 2004.

2. F. Bacchus and F. Kabanza. Planning for temporally extended goals. in Annals of Mathematics and AI, 22:5-27, 1998. 
3. M. Benerecetti, F. Giunchiglia and L. Serafini. Model Checking Multiagent Systems. Journal of Logic and Computation. Special Issue on Computational Aspects of MultiAgent Systems, 8(3):401-423. 1998.

4. R. Bordini, M. Fisher, C. Pardavila and M. Wooldridge. Model Checking AgentSpeak. AAMAS 2003, pp. 409-416, 2003.

5. D. Calvanese, G. De Giacomo and M.Y.Vardi. Reasoning about Actions and Planning in LTL Action Theories. In Proc. KR'02, 2002.

6. FIPA Contract Net Interaction Protocol Specification, 2002. Available at http://www.fipa.org.

7. N. Fornara and M. Colombetti. Defining Interaction Protocols using a Commitmentbased Agent Communication Language. Proc. AAMAS'03, Melbourne, pp. 520-527, 2003.

8. R. Gerth, D. Peled, M.Y.Vardi and P. Wolper. Simple On-the-fly Automatic verification of Linear Temporal Logic. In Proc. 15th Work. Protocol Specification, Testing and Verification, Warsaw, June 1995, North Holland.

9. L. Giordano and A. Martelli. On-the-fly Automata Construction for Dynamic Linear Time Temporal Logic. TIME 04, June 2004.

10. L. Giordano, A. Martelli, and C. Schwind. Reasoning About Actions in Dynamic Linear Time Temporal Logic. In FAPR'00 - Int. Conf. on Pure and Applied Practical Reasoning, London, September 2000. Also in The Logic Journal of the IGPL, Vol. 9, No. 2, pp. 289-303, March 2001.

11. L. Giordano, A. Martelli, and C. Schwind. Specifying and Verifying Systems of Communicating Agents in a Temporal Action Logic. In Proc. AI*IA'03, Pisa, pp. 262-274, Springer LNAI 2829, September 2003.

12. F. Giunchiglia and P. Traverso. Planning as Model Checking. In Proc. The 5th European Conf. on Planning (ECP'99), pp.1-20, Durham (UK), 1999.

13. F. Guerin. Specifying Agent Communication Languages. PhD Thesis, Imperial College, London, April 2002.

14. F. Guerin and J. Pitt. Verification and Compliance Testing. Communications in Multiagent Systems, Springer LNAI 2650, pp. 98-112, 2003.

15. J.G. Henriksen and P.S. Thiagarajan. Dynamic Linear Time Temporal Logic. in Annals of Pure and Applied logic, vol.96, n.1-3, pp.187-207, 1999

16. M.P. Huget and M. Wooldridge. Model Checking for ACL Compliance Verification. ACL 2003, Springer LNCS 2922, pp. 75-90, 2003.

17. M.Pistore and P.Traverso. Planning as Model Checking for Extended Goals in Non-deterministic Domains. Proc. IJCAI'01, Seattle, pp.479-484, 2001.

18. R. Reiter. The frame problem in the situation calculus: a simple solution (sometimes) and a completeness result for goal regression. In Artificial Intelligence and Mathematical Theory of Computation: Papers in Honor of John McCarthy, V. Lifschitz, ed.,pages 359-380, Academic Press, 1991.

19. M. P. Singh. A social semantics for Agent Communication Languages. In IJCAI-98 Workshop on Agent Communication Languages, Springer, Berlin, 2000.

20. M. Wooldridge. Semantic Issues in the Verification of Agent Communication Languages. Autonomous Agents and Multi-Agent Systems, vol. 3, pp. 9-31, 2000.

21. M. Wooldridge, M. Fisher, M.P. Huget and S. Parsons. Model Checking MultiAgent Systems with MABLE. In $A A M A S^{\prime}$ '02, pp. 952-959, Bologna, Italy, 2002.

22. P. Yolum and M.P. Singh. Flexible Protocol Specification and Execution: Applying Event Calculus Planning using Commitments. In $A A M A S^{\prime}$ 02, pp. 527-534, Bologna, Italy, 2002. 